\title{
distinct: a novel approach to differential distribution analyses
}

\author{
Simone Tiberi ${ }^{1 *}$, Helena L Crowell ${ }^{1}$, Lukas M Weber ${ }^{2}$, Pantelis Samartsidis $^{3}$ and Mark D Robinson ${ }^{1}$ \\ ${ }^{1}$ Department of Molecular Life Sciences and Swiss Institute of Bioinformatics, University of Zurich, \\ Zurich, Switzerland. \\ ${ }^{2}$ Department of Biostatistics, Johns Hopkins Bloomberg School of Public Health, Baltimore, MD, USA. \\ ${ }^{3}$ MRC Biostatistics Unit, School of Clinical Medicine, University of Cambridge, Cambridge, UK. \\ * e-mail: Simone.Tiberi@uzh.ch
}

\section{Abstract}

2 We present distinct, a general method for dif3 ferential analysis of full distributions that is 4 well suited to applications on single-cell data, 5 such as single-cell RNA sequencing and high6 dimensional flow or mass cytometry data. High7 throughput single-cell data reveal an unprece8 dented view of cell identity and allow com9 plex variations between conditions to be discov10 ered; nonetheless, most methods for differential 11 expression target differences in the mean and 12 struggle to identify changes where the mean is 13 only marginally affected. distinct is based on 14 a hierarchical non-parametric permutation ap15 proach and, by comparing empirical cumulative 16 distribution functions, identifies both differen17 tial patterns involving changes in the mean, as 18 well as more subtle variations that do not in19 volve the mean. We performed extensive bench20 marks across both simulated and experimen21 tal datasets from single-cell RNA sequencing 22 and mass cytometry data, where distinct shows 23 favourable performance, identifies more differ24 ential patterns than competitors, and displays 25 good control of false positive and false discovery 26 rates. distinct is available as a Bioconductor $\mathbf{R}$ 27 package.

28 keywords: Differential distribution; Differential anal29 yses; Differential state; High-throughput single-cell so data; Single-cell RNA-seq; Single-cell flow and mass cy31 tometry; Permutation tests.

\section{${ }_{32}$ Background}

33 Technology developments in the last decade have led to 34 an explosion of high-throughput single-cell data, such 35 as single-cell RNA sequencing (scRNA-seq) and high36 dimensional flow or mass cytometry data, allowing re-
37 searchers to investigate biological mechanisms at single38 cell resolution. Single-cell data have also extended the 39 canonical definition of differential expression by dis40 playing cell-type specific responses across conditions, 41 known as differential state (DS) [28], where genes or 42 proteins vary in specific sub-populations of cells (e.g., 43 a cytokine response in myeloid cells but not in other 44 leukocytes 10 ). Classical bulk differential expression 45 methods have been shown to perform well when used 46 on single-cell measurements $22,23,27$ and on aggre47 gated data (i.e., averages or sums across cells), also re48 ferred to as pseudo-bulk (PB) [5, 28]. However, most 49 bulk and PB tools focus on shifts in the means, and 50 may conceal information about cell-to-cell heterogene51 ity. Indeed, single-cell data can show more complex 52 variations (Figure 1 and Supplementary Figure 1); such 53 patterns can arise due to increased stochasticity and 54 heterogeneity, for example owing to oscillatory and un55 synchronized gene expression between cells, or when 56 some cells respond differently to a treatment than oth57 ers [12,27]. In addition to bulk and PB tools, other 58 methods were specifically proposed to perform differ59 ential analyses on single-cell data (notably: $s c D D$ [12], 60 SCDE [11], MAST [8], BASiCS [26] and mixed mod61 els $[24 \mid)$. Nevertheless, they all present significant limi62 tations: BASiCS does not perform cell-type specific dif63 ferential testing between conditions, scDD does not di64 rectly handle covariates and biological replicates, while ${ }_{65} \mathrm{~PB}, \mathrm{SCDE}, \mathrm{MAST}$ and mixed models performed poorly ${ }_{66}$ in previous benchmarks when detecting differential pat67 terns that do not involve the mean [5, 12].

\section{${ }_{68}$ Results}

\section{9 distinct's full distribution approach}

70 To overcome these challenges, we developed distinct, a 71 flexible and general statistical methodology to perform 72 differential analyses between groups of distributions. 
73 distinct is particularly suitable to compare groups of 120 non-parametric permutation approach (see Methods). 74 samples (i.e., biological replicates) on single-cell data.

75 Our approach computes the empirical cumulative dis76 tribution function (ECDF) from the individual (e.g., 77 single-cell) measurements of each sample, and compares 78 ECDFs to identify changes between full distributions, 79 even when the mean is unchanged or marginally in80 volved (Figure 1 and Supplementary Figure 1). First, 81 we compute the ECDF of each individual sample; then 82 we build a fine grid and, at each cut-off, we average the 83 ECDFs within each group, and compute the absolute 84 difference between such averages. A test statistic, $s^{o b s}$, 85 is obtained by adding these absolute differences.

86 More formally, assume we are interested in compar87 ing two groups, that we call $A$ and $B$, for which $N_{A}$ 88 and $N_{B}$ samples are available, respectively. The ECDF 89 for the $i$-th sample in the $j$-th group, is denoted by 90 $e c d f_{i}^{(j)}($.$) , for j \in\{A, B\}$ and $i=1, \ldots, N_{j}$. We 91 then define $K$ equally spaced cut-offs between the mini92 mum, min, and maximum, max, values observed across 93 all samples: $b_{1}, \ldots, b_{K}$, where $b_{k}=\min +k \times l$, for $94 k=1, \ldots, K$, with $l=(\max -\min ) /(K+1)$ being 95 the distance between two consecutive cut-offs. We ex96 clude min and $\max$ from the cut-offs because, trivially, ${ }_{97} e c d f_{i}^{(j)}(\min )=0$ and $e c d f_{i}^{(j)}(\max )=1, \forall j, i$. At ev98 ery cut-off, we compute the absolute difference between 99 the mean ECDF in the two groups; our test statistic, $100 s^{o b s}$, is obtained by adding these differences across all 101 cut-offs:

$$
s^{o b s}=\sum_{k=1}^{K}\left|\frac{\sum_{i=1}^{N_{A}} e c d f_{i}^{(A)}\left(b_{k}\right)}{N_{A}}-\frac{\sum_{i=1}^{N_{B}} e c d f_{i}^{(B)}\left(b_{k}\right)}{N_{B}}\right| .
$$

102 Note that in differential state analyses, these operations 103 are repeated for every gene-cluster combination.

104 Intuitively, $s^{o b s}$, which ranges in $[0, \infty)$, approximates 105 the area between the average ECDFs, and represents 106 a measure of distance between two groups of densities: 107 the bigger $s^{o b s}$, the greater the distance between groups. 108 The number of cut-offs $K$, which can be defined by 109 users, is set to 25 by default, because no detectable 110 difference in performance was observed when further 111 increasing it (data not shown). Note that, although at 112 each cut-off we compute the average across each group's 113 curves, ECDFs are computed separately for each indi114 vidual sample, therefore our approach still accounts for 115 the within-group variability; indeed, at a given thresh116 old, the average of the sample-specific ECDFs differs 117 from the group-level ECDF (i.e., the curve based on 118 all individual measurements from the group). The null 168 We conducted an extensive benchmark, based on 119 distribution of $s^{o b s}$ is then estimated via a hierarchical 169 scRNA-seq and mass cytometry simulated and experi123 few permutations are available from small samples. We 124 overcome this by permuting cells, which is still pos125 sible in small samples, because there are many more 126 cells than samples. In principle, this may lead to an 127 inflation of false positives due to lack of exchangabil128 ity (see Methods); nonetheless, in our analyses, distinct 129 provides good control of both false positive and false 130 discovery rates.

131 Importantly, distinct is general and flexible: it targets 132 complex changes between groups, explicitly models bio133 logical replicates within a hierarchical framework, does 134 not rely on asymptotic theory, avoids parametric as135 sumptions, and can be applied to arbitrary types of 136 data. Additionally, distinct can also adjust for sample137 level cell-cluster specific covariates (i.e., whose effect 138 varies across cell clusters), such as batch effects,: dis139 tinct fits a linear model with the input data (e.g., CPMs 140 or $\log 2-\mathrm{CPMs}$ ) as response variable, and the covariates 141 as predictors; the method then removes the estimated 142 effect of covariates, and performs differential testing on 143 these normalized values (see Methods).

144 Furthermore, to enhance the interpretability of differen145 tial results, distinct provides functionalities to compute 146 ( $\log$ ) fold changes between conditions, and to plot den147 sities and ECDFs, both for individual samples and at 148 the group-level.

149 Note that, although distinct and the Kolmogorov150 Smirnov [15] (KS) test share similarities (they both 151 compare distributions via non-parametric tests), the 152 two approaches present several conceptual differences. 153 Firstly, the KS considers the maximum distance be154 tween two ECDFs, while our approach estimates the 155 overall distance between ECDFs, which in our view is 156 a more appropriate way to measure the difference be157 tween distributions. Secondly, the KS test only com158 pares two individual densities, while our framework 159 compares groups of distributions. Thirdly, while the $160 \mathrm{KS}$ statistic relies on asymptotic theory, our framework 161 uses a permutation test. Finally, a comparison between 162 distinct and $s c D D[12$ based on the KS test (labelled $163 s c D D-K S$ ) shows that our method, compared to the KS 164 test, has greater statistical power to detect differential 165 effects and leads to fewer false discoveries (see Simula166 tion studies).

\section{${ }_{167}$ Simulation studies} 121 A major disadvantage of permutation tests, which of122 ten restricts its usage on biological data, is that too 
bioRxiv preprint doi: https://doi.org/10.1101/2020.11.24.394213; this version posted May 6, 2021. The copyright holder for this preprint (which was not certified by peer review) is the author/funder, who has granted bioRxiv a license to display the preprint in perpetuity. It is made available under aCC-BY 4.0 International license.

DE

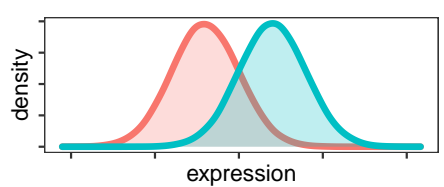

DP

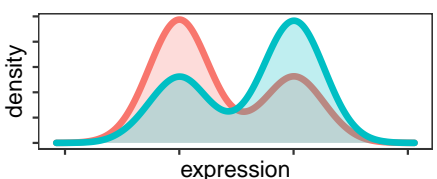

expression

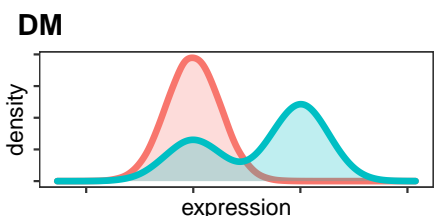

expression

DB

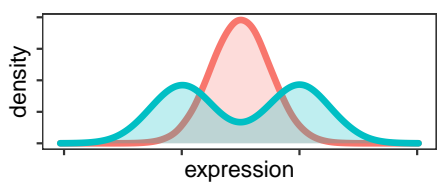

expression

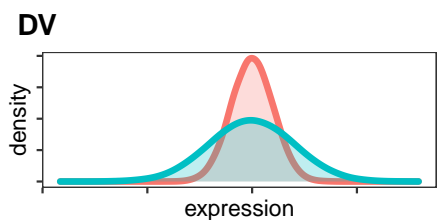

expression

group $=\mathrm{A}=\mathrm{B}$

Figure 1: Cumulative distribution functions (CDFs) unravel differences between distributions. Density (left panels) and CDF (right panels) of five differential patterns: differential variability (DV), and the four proposed by Korthauer et. al. [12]: differential expression (DE), differential proportion (DP), differential modality (DM), and both differential modality and different component means (DB).

170 mental datasets to investigate distinct's ability to iden171 tify differential patterns in sub-populations of cells.

172 First, we simulated droplet scRNA-seq data via mus173 cat [5] (see Methods). We ran five simulation repli174 cates for each of the differential profiles in Figure 1, 190 We considered three different normalizations: counts 175 with $10 \%$ of the genes being differential in each clus- 191 per million (CPMs), logarithm of CPMs to base 2 (log2176 ter, where DE (differential expression) indicates a shift $192 \mathrm{CPMs}$ ) and residuals from variance stabilizing normal177 in the entire distribution, DP (differential proportion) 193 ization from sctransform (vstresiduals) [9]. We com178 implies two mixture distributions with different propor- 194 pared distinct to several PB approaches from muscat, 179 tions of the two components, DM (differential modal- 195 based on edgeR [21], limma-voom and limma-trend [20], 180 ity) assumes a unimodal and a bimodal distribution, 196 which emerged among the best performing methods for $181 \mathrm{DB}$ (both differential modality and different component 197 differential analyses from scRNA-seq data [5, 23]. We 182 means) compares a unimodal and a bimodal distribu- 198 further considered three methods from muscat based 183 tion with the same overall mean, and DV (differential 199 on mixed models (MM), namely $M M$-dream2, $M M$ 184 variability) refers to two unimodal distributions with 200 vstresiduals and MM-nbinom (see Methods). Finally, 185 the same mean but different variance (Figure 1 and 201 we included $s c D D[12$, which is conceptually similar 186 Supplementary Figure 1). Each individual simulation 202 to our approach: scDD implements a non-parametric 187 consists of 4,000 genes, 3,600 cells, separated into 3 clus- 203 method to detect changes between individual distri188 ters, and two groups of 3 samples each, corresponding 204 butions from scRNA-seq, based on the Kolmogorov189 to an average of 200 cells per sample in each cluster. $\quad 205$ Smirnov test, $s c D D-K S$, and on a permutation ap-

DE
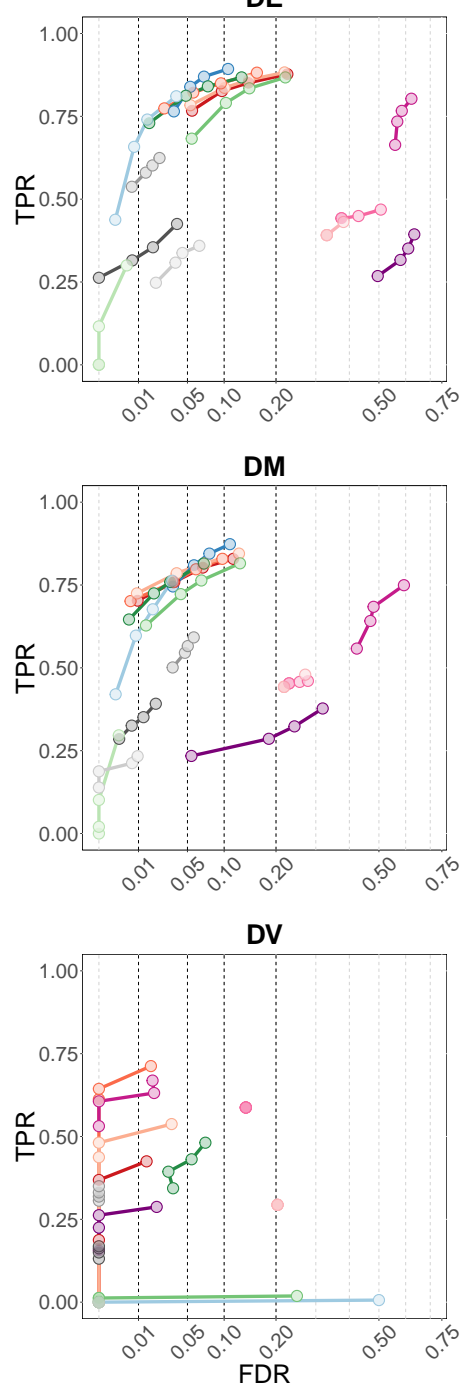

Figure 2: distinct identifies various differential patterns and controls for the FDR. TPR vs. FDR in muscat simulated data in Figure 1. Results are averages across the five simulation replicates. Circles indicate observed FDR for $0.01,0.05,0.1$ and 0.2 significance thresholds. Two groups of 3 samples are compared and, on average, 200 cells are available for every sample in each of three clusters. 
bioRxiv preprint doi: https://doi.org/10.1101/2020.11.24.394213; this version posted May 6, 2021. The copyright holder for this preprint (which was not certified by peer review) is the author/funder, who has granted bioRxiv a license to display the preprint in perpetuity. It is made available under aCC-BY 4.0 International license.

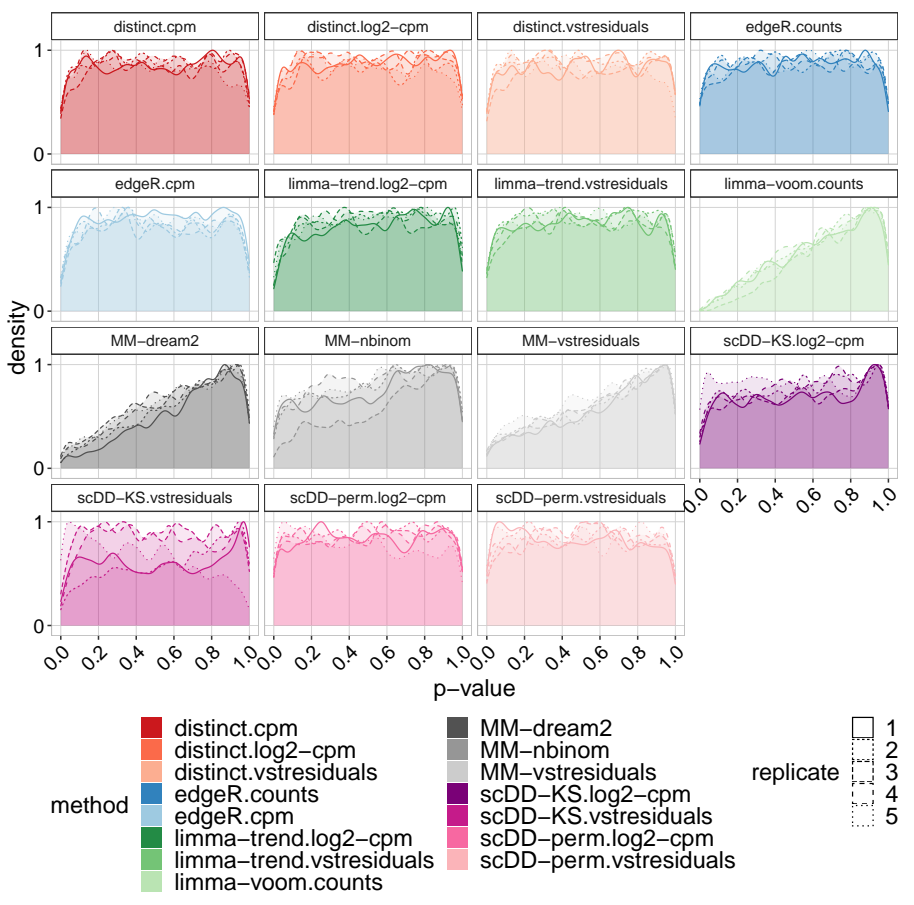

Figure 3: distinct has uniform null p-values. Density of raw p-values in muscat null simulated data; each replicate represents a different null simulation. Two groups of 3 samples are compared and, on average, 200 cells are available for every sample in each of three clusters.

206 proach, scDD-perm. For $s c D D$-perm we used 100 per207 mutations to reduce the computational burden.

208 In all scenarios and on all three input datasets, dis209 tinct shows favourable performance: it has good sta210 tistical power while controlling for the false discov211 ery rate (FDR) (Figure 2). In particular, for DE, ${ }_{212} \mathrm{DP}$ and DM, distinct has similar performance to the 213 best performing competitors (edgeR.counts and limma214 trend.log2-CPMs), while for DB and DV, it achieves 215 significantly higher true positive rate (TPR), especially 216 when using log2-CPMs. PB methods in general per217 form well for differential patterns involving changes in 218 the mean (DE, DP and DM), but struggle to identify $219 \mathrm{DB}$ and DV patterns. $s c D D$ provides good TPR across 220 all patterns when using the KS test on vstresiduals 221 (scDD-KS.vstresiduals), while the TPR is significantly 222 reduced when using log2-CPMs and with the permu223 tation approach $(s c D D$-perm $)$; however, $s c D D$ methods 224 also show a significant inflation of the FDR. In contrast, $225 \mathrm{MM}$ methods provide good control of the FDR but have 226 low statistical power in all differential scenarios.

227 We further simulated five null simulation replicates 228 with no differential patterns; again with each simula229 tion having 4,000 genes, 3,600 cells, 3 cell clusters and 230 two groups of 3 samples each. In the null simulated 231 data, no method presents an inflation of false positives,

232 with distinct, edgeR, limma-trend and $s c D D$ showing 250 From a computational perspective, distinct required

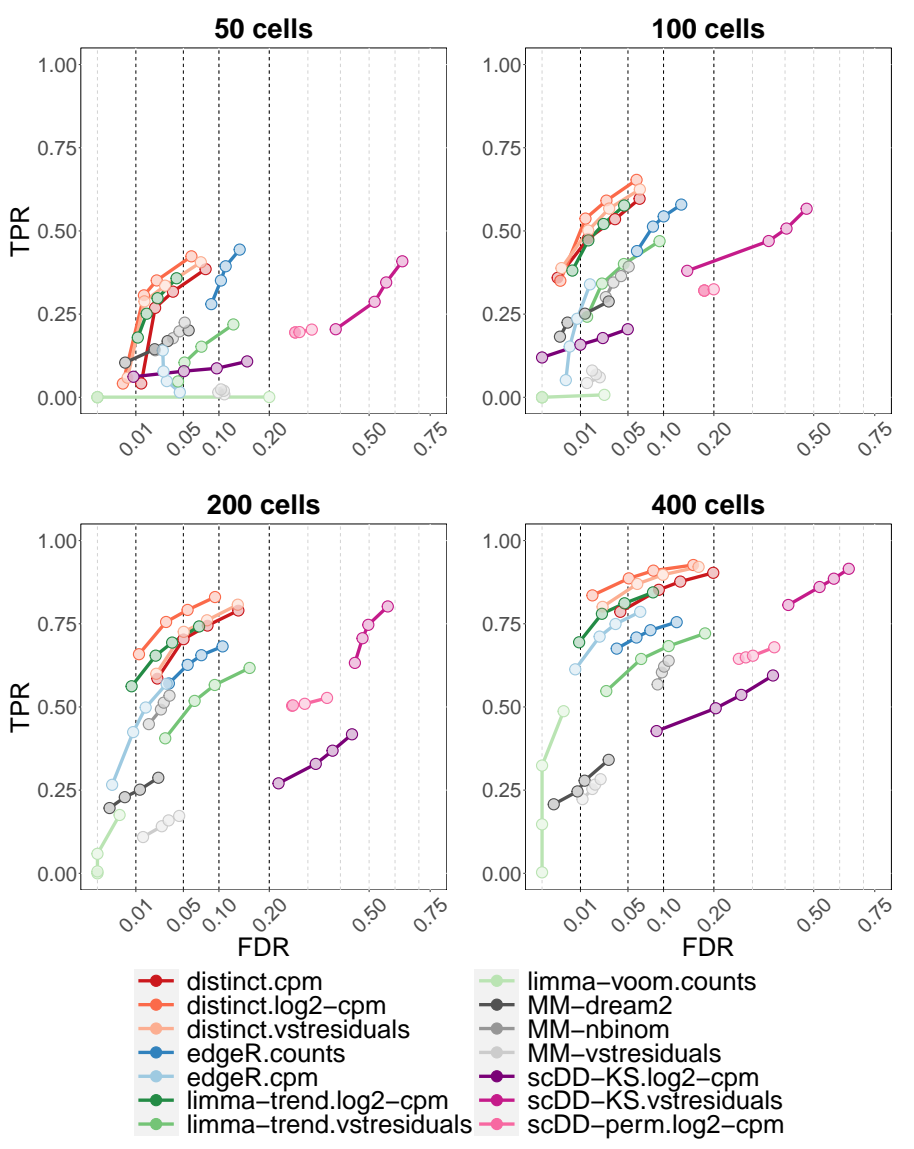

Figure 4: distinct achieves good performance when varying the number of available cells. TPR vs. FDR in muscat simulated data; with 50,100, 200 and 400 cells per cluster-sample combination, corresponding to a total of $900,1,800,3,600$ and 7,200 cells, respectively. Results are aggregated over the five replicate simulations of each differential type (DE, DP, DM, DB and DV), contributing in equal fraction. Each individual simulation replicate consists of 4,000 genes, 3 cell clusters and two groups of 3 samples each. Circles indicate observed FDR for 0.01, 0.05, 0.1 and 0.2 significance thresholds. Note that $s c D D$-perm.vstresiduals was excluded from this analysis due to its computational cost.

233 approximately uniform p-values for all types of input 234 data (Figure 3).

235 We also extended previous simulations to add a cell236 type specific batch effect (i.e., a batch effect that affects 237 differently each cell-type) [5, 14]. In particular, we sim238 ulated 2 batches, that we call $b_{1}$ and $b_{2}$, with one group 239 of samples having two samples associated to $b_{1}$ and one 240 to $b_{2}$, and the other group of samples having two sam241 ples from batch $b_{2}$ and one from $b_{1}$. Differential results 242 are substantially unchanged (Supplementary Figure 2), 243 which shows distinct can effectively remove nuisance 244 confounders. Furthermore, by varying the number of 245 cells in the simulated data, we show that, compared to $246 \mathrm{~PB}, \mathrm{MM}$ and $s c D D$ methods, distinct achieves higher 247 overall TPR, while controlling for the FDR, regardless 248 of the number of available cells (Figure 5 and Supple249 mentary Figure 3). 

available under aCC-BY 4.0 International license.

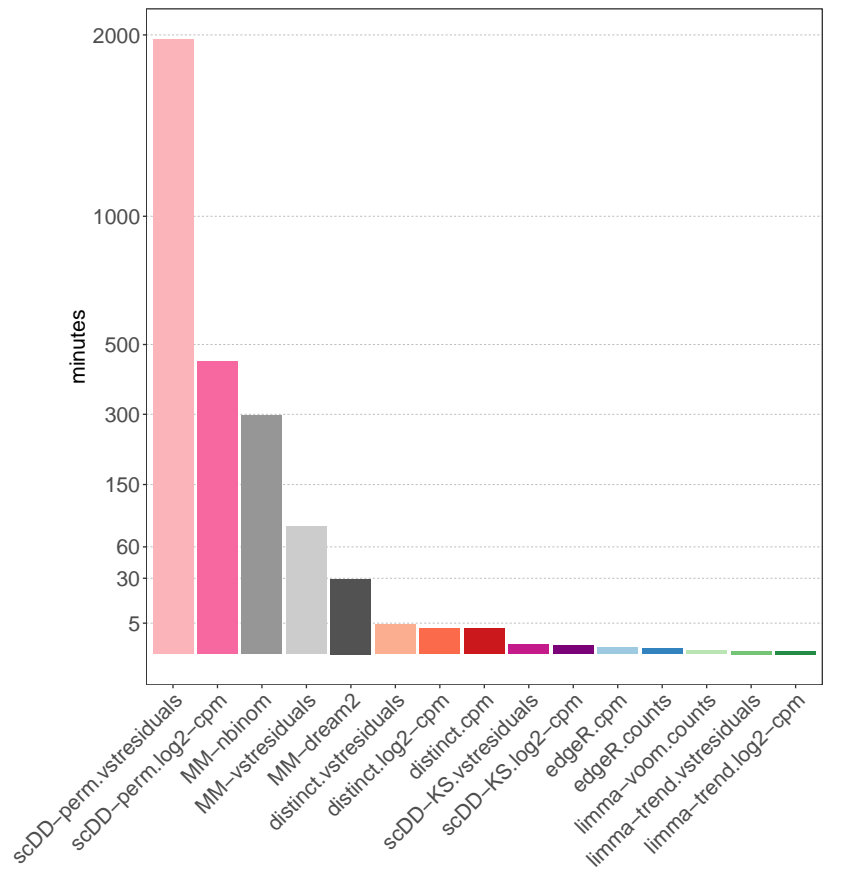

Figure 5: distinct requires more computational resources than PB and $s c D D-K S$ methods, but significantly less than MM and scDD-perm models. Average computing time, expressed in minutes, in muscat main simulations (Figures 2-3). For each method, times are averaged across simulation types (DE, DP, DM, DB, DV and null) and, for each type, across the five replicate simulations; in each replicate 3,600 cells are available (200, on average, per cluster-sample combination). distinct, MM and $s c D D$ models were run on 3 cores, while pseudo-bulk methods based on edgeR and limma used a single core because they do not allow for parellel computing.

251 an average time of 3.4 to 4.5 minutes per simulation, 252 which is higher than $\mathrm{PB}$ methods ( 0.1 to 0.2 minutes) 253 and $s c D D-K S$ (0.4 to 0.5 minutes), but significantly 254 lower than MM approaches (29.4 to 297.3 minutes) and 255 scDD-perm (447.5 to 1970.1 minutes) (Figure 4 and 256 Supplementary Table 1). All methods were run on 3 257 cores, except PB approaches, which used a single core, 258 because they do not allow for parellel computing.

259 We further considered the semi-simulated mass cytom260 etry data from Weber et al. [28] (labelled diffcyt sim261 ulation), where spike-in signals were computationally 262 introduced in experimental data [3], hence maintain- 275 separate approaches (see Methods): i) similarly to the 263 ing the properties of real biological data while also 276 muscat simulation study, cell labels were defined based 264 embedding a known ground truth signal. We evalu- 277 on 8 manually annotated cell types [28] (Figure 6a), 265 ated distinct and two methods from diffcyt, based on 278 and ii) as in the original diffcyt study from Weber et 266 limma [20] and linear mixed models (LMM), which out- 279 al. 28], cells were grouped into 100 high-resolution clus267 performed competitors on these same data [28]. In 280 ters (based on 10 cell-type markers, see Methods) via 268 particular, we considered three datasets from Weber 281 unsupervised clustering (Figure 6b). In the main simu269 et al. [28]: the main DS dataset and two more where 282 lation, distinct achieves higher TPR when considering 270 differential effects were diluted by 50 and 75\%. Each 283 cell-type labels (Figure 6a, 'main'), while all methods 271 dataset consists of 24 protein markers, 88,435 cells, and 284 exhibit substantially overlapping performance when us272 two groups (with and without spike-in signal) of 8 sam- 285 ing unsupervised clustering (Figure 6b, 'main'). In both 273 ples each. Measurements were first transformed, and 286 clustering approaches, as the magnitude of the differ274 then cells were grouped into sub-populations with two 287 ential effect decreases, the distance between methods 
288 increases: diffcyt tools show a significant drop in the 289 true positive rate (TPR) whereas distinct maintains a 290 higher TPR while effectively controlling for the false 291 discovery rate (FDR) (Figures 6a-b and Supplemen292 tary Figure 4). This indicates that distinct has good 293 statistical power to detect even small changes between 294 conditions. We also considered the three replicate null 295 datasets from Weber et al. 28 (i.e., with no differential 296 effect), containing 24 protein markers and 88,438 cells 297 across 8 cell types, and found that all methods display 298 approximately uniform p-values (Figure 6c).

\section{Experimental data analyses}

300 In order to investigate false positive rates (FPRs) in 301 real data, we considered two experimental scRNA-seq 302 datasets where no differential signals were expected, by 303 comparing samples from the same experimental con304 dition. Given the high computational cost and low 305 power of MM, and the high FDR of $s c D D$ models, for 306 the real data analyses, we only included distinct and 307 PB methods. We considered gene-cluster combinations 308 with at least 20 non-zero cells across all samples. The 309 first dataset (labelled T-cells) consists of a Smart-seq2 310 scRNA-seq dataset of 23,459 genes and 11,138 T cells 311 isolated from peripheral blood from 12 colorectal can312 cer patients 30$]$. We automatically separated cells in 31311 clusters (via igraph [1, 6]), and generated replicate 314 datasets, by randomly separating, three times, the 12 315 patients to two groups of size 6 . The second dataset 316 (labelled Kang) contains 10x droplet-based scRNA-seq 317 peripheral blood mononuclear cell data from 8 Lupus 318 patients, before (controls) and after (stimulated) 6h319 treatment with interferon- $\beta$ (INF- $\beta$ ), a cytokine known 320 to alter the transcriptional profile of immune cells 10 . 321 The full dataset contains 35,635 genes and 29,065 cells, 322 which are separated (via manual annotation [10]) into 3238 cell types. One of the 8 patients was removed as it 324 appears to be a potential outlier (Supplementary Fig325 ures 5-7). Here we only included singlet cells and cells 326 assigned to a cell population, and considered control 327 samples only, resulting in 11,854 cells. Again, we ar328 tificially created three replicate datasets by randomly 329 assigning the 7 retained control samples in two groups 330 of size 3 and 4 . In both null analyses, we found that 331 limma-trend leads to a major increase of FPRs, dis332 tinct's p-values are only marginally inflated towards 0 , 333 while edgeR and limma-voom are the most conservative 334 methods and provide the best control of FPRs (Figure $3357 \mathrm{a}$ and Supplementary Tables 2-3).

336 We then considered again the Kang dataset, and per337 formed a DS analysis between controls and stimulated 339 tient, and only considered singlet cells and cells as338 samples. Again, we removed one potential outlier pa- 340 signed to a cell population, resulting in 35,635 genes, a
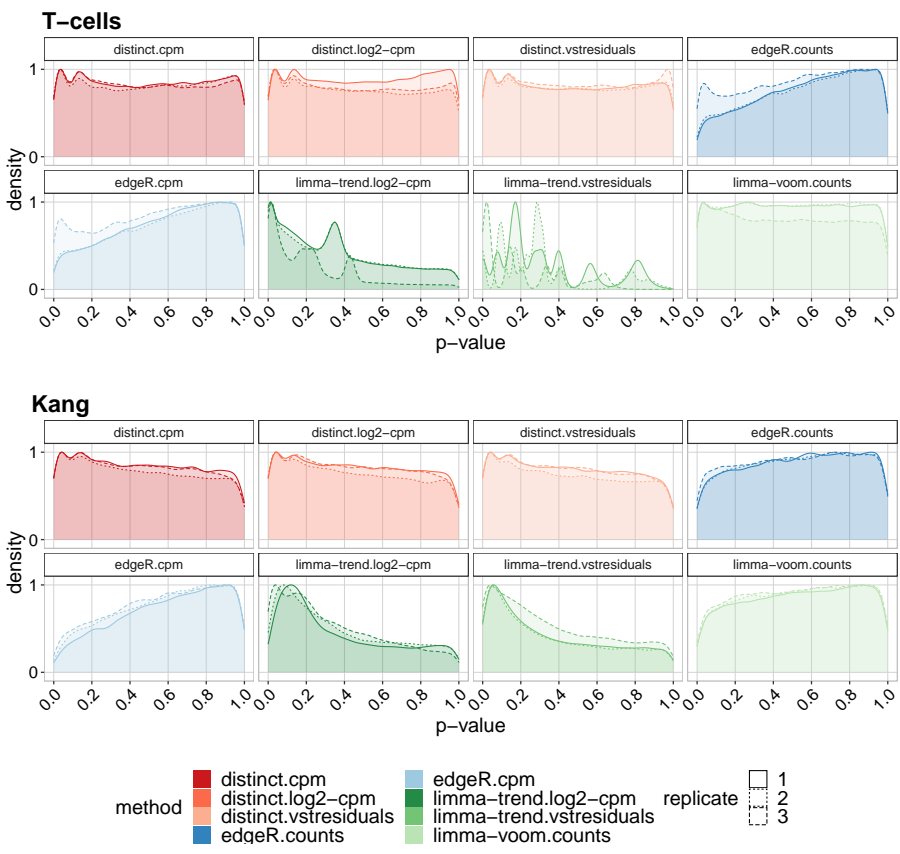

b
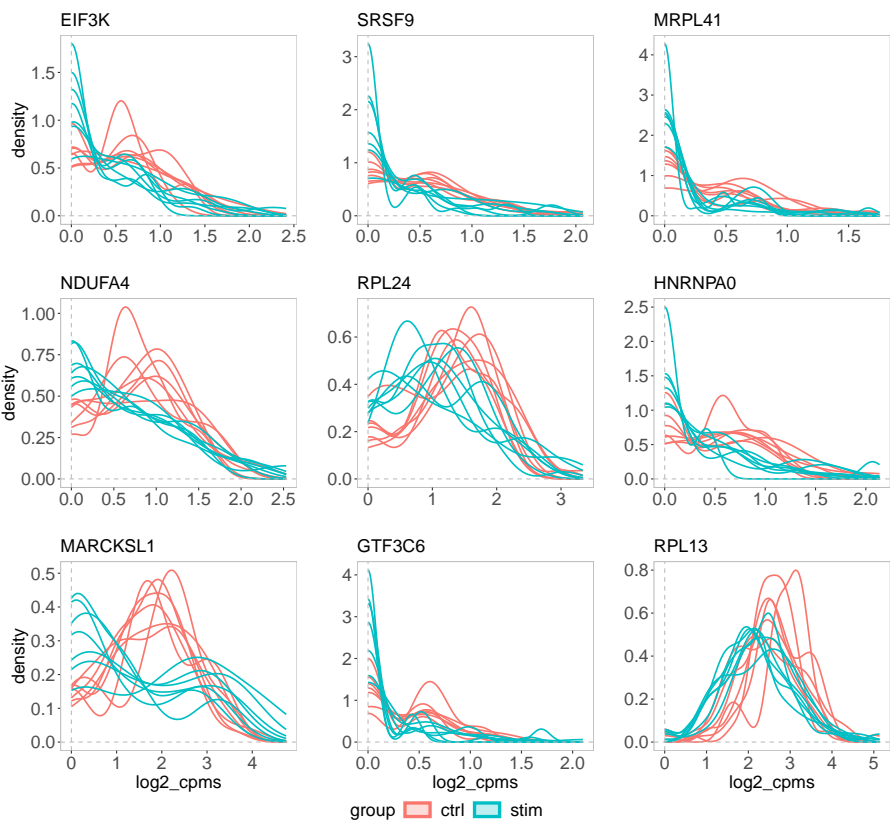

Figure 7: On experimental scRNA-seq data, distinct discovers non-canonical differential patterns, and has almost-uniform null p-values. (a) Density of raw p-values in the null T-cells (top) and Kang (bottom) experimental data. Each replicate represents a random partition of samples in two groups. The $T$-cells data consists of 12 samples and 11,138 cells across 11 clusters. For the Kang dataset, we retained 7 samples and 11,854 cells across 8 clusters. (b) Density of log2-CPMs for nine examples of differential patterns identified by distinct on all input data (adjusted p-values $<$ 0.05 ), and not by any PB tool (adjusted p-values $>0.05$ ), on the Kang dataset when comparing controls and stimulated samples. Gene RPL13 was identified in FCGR3A+ Monocytes cells, while all other genes were detected in Dendritic cells. Each line represents a sample. 
34123,571 cells across 8 cell types and 14 samples; we fur- 392 to various types of data, beyond the single-cell applica342 ther filtered gene-cluster combinations with less than 20393 tions shown here.

343 non-zero cells across all samples. We found that distinct 344 identifies more differential patterns than PB methods, 345 with edgeR and limma-voom being the most conser346 vative methods, and that its results are very coherent 347 across different input data (Supplementary Figure 8). 348 When visually investigating the gene-cluster combina349 tions detected by distinct (adjusted p-value $<0.05$ ), on 350 all input data (CPMs, $\log 2-\mathrm{CPMs}$ and vstresiduals), 351 and not detected by any PB method (adjusted p-value $352>0.05$ ), we found several interesting non-canonical dif353 ferential patterns (Figure 7b and Supplementary Fig354 ures 9-17). In particular, gene MARCKSL1 displays 355 a DB pattern, with stimulated samples having higher 356 density on the tails and lower in the centre of the dis357 tribution, gene RPL13 mirrors classical DE, while the 358 other genes seem to emulate DP profiles. Interestingly, 359 eight out of nine of these genes are known tumor prog360 nostic markers: EIF3K for cervical and renal cancer, 361 SRSF9 for liver cancer and melanoma, NDUFA4 for 362 renal cancer, RPL24 for renal and thyroid cancer, HN363 RNPA0 for renal and pancreatic cancer, MARCKSL1 364 for liver and renal cancer, GTF3C6 for liver cancer and 365 RPL13 for endometrial and renal cancer [25]. This is 366 an interesting association, considering that INF- $\beta$ stim367 ulation is known to inhibit and interfere with tumor 368 progression [7, 19]. Finally, Supplementary Figures 936917 show how distinct can identify differences between 370 groups of distributions even when only a portion of the 371 ECDF varies between conditions.

\section{${ }_{372}$ Discussion}

373 High-throughput single-cell data can display complex 374 differential patterns; nonetheless, most methods for dif375 ferential expression fail to identify changes where the 376 mean is not affected. To overcome present limitations, 377 we have introduced distinct, a general method to iden378 tify differential patterns between groups of distribu379 tions, which is particularly well suited to perform differ380 ential analyses on high-throughput single-cell data. We 381 ran extensive benchmarks on both simulated and ex382 perimental datasets from scRNA-seq and mass cytom383 etry data, where our method exhibits favourable per384 formance, provides good control of the FPR and FDR, 385 and is able to identify more patterns of differential ex- 428 In order to test for differences between groups, we em386 pression compared to canonical tools, even when the 429 ploy a hierarchical permutation approach: to estimate 387 overall mean is unchanged. Furthermore, distinct al- 430 the null distribution of $s^{\text {obs }}$, we permute the individual 388 lows for biological replicates, can adjust for covariates 431 observations (e.g., single-cell measurements) instead of 389 (e.g., batch effects), and does not rely on asymptotic 432 the samples. Note that this violates the exchangeability 390 theory. Finally, note that distinct is a very general test 433 assumption of permutation tests and, hence, p-values 391 that, due to its non-parametric nature, can be applied 434 are not guaranteed to be uniformly distributed under 
435 the null hypothesis; nonetheless, in our simulated and 471 package 9 (except for the T-cells data, where, due to 436 experimental analyses, we empirically show that dis- 472 a failure of sctransform's variance stabilizing normal437 tinct provides good control of both false positive and 473 ization, we used DESeq2's vst transformation [13]). 438 false discovery rates. We randomly permute individual 439 observations $P$ times across all samples and groups, by 440 retaining the original sample sizes. We denote by $s_{p}$ 441 the test statistic computed at the $p$-th permutation, $442 p=1, \ldots, P$. A p-value, $\tilde{p}$, is obtained as $[18$ :

$$
\tilde{p}=\frac{\sum_{p=1}^{P} \mathbf{1}\left(s_{p} \geq s^{o b s}\right)+1}{P+1},
$$

443 where $\mathbf{1}$ (cond) is 1 if cond is true, and 0 otherwise. In 444 order to accurately infer small p-values, when $\tilde{p}$ is below 445 some pre-defined thresholds, the number of permuta446 tions are automatically increased and $\tilde{p}$ is re-computed. 447 By default, distinct initially computes 100 permuta448 tions; when $\tilde{p} \leq 0.1$ these are increased to 500 ; when 449 the new $\tilde{p} \leq 0.01$ we use 2,000 permutations, which 450 are further increased to 10,000 if $\tilde{p} \leq 0.001$. Note that 451 the number of permutations (i.e., 100, 500, 2,000 and 452 10,000) can be specified by the user.

\section{${ }_{453}$ Covariates}

474 In mass cytometry datasets, measurements were trans475 formed via diffcyt's transformData function, which ap476 plies an arcsinh transformation.

\section{7 diffcyt simulation}

478 The diffcyt semi-simulated data originates from a real 479 mass cytometry dataset of healthy peripheral blood 480 mononuclear cells from two paired groups of 8 samples 481 each [3]; one group contains unstimulated cells, while 482 the other was stimulated with B cell receptor/Fc recep483 tor cross-linker. The original dataset contains a total 484 of 172,791 cells and 24 protein markers: 10 of these 485 are cell-type markers used for cell clustering, while 14 486 are cell state markers used for differential state anal$487 \mathrm{yses}$; the distinction between cell state and cell-type 488 markers is based on prior biological knowledge [28]. 489 In Weber et al. 28, semi-simulated data were gener490 ated by separating the cells of each unstimulated sam491 ple in two artificial samples; a differential signal was 492 then computationally introduced by replacing, in one Assume we observe $Z$ nuisance covariates, and that $N_{493}$ group, unstimulated B cells with B cells from stimusamples are available across all groups, where for the 494 lated samples. Measurements were transformed and $i$-th sample we observe $C_{i}$ values (e.g., single-cell measurements). We fit the following linear model:

$$
y_{c}^{(i)}=\beta_{0}+\sum_{z=1}^{Z} \beta_{z} X_{z}^{(i)}+\epsilon_{c}^{(i)}, \text { for } i=1, \ldots, N,
$$

$$
\text { and } c=1, \ldots, C_{i} \text {, }
$$

495 cells clustered via diffcyt's transformData (which ap496 plies an arcsinh transformation) and generateClusters 497 functions, respectively. For the DS simulation in Fig498 ure 6b, as in Weber et al. [28], we evaluated methods' 499 performance in terms of detecting DS for phosphory500 lated ribosomal protein $\mathrm{S} 6$ (pS6) in B cells, which is 501 the strongest differential signal across the cell types in 502 this dataset [17, 28]. For the DS simulation in Figure 454 where $y_{c}^{(i)}$ represents the $c$-th observation for the $i$-th ${ }_{503}^{502} 6 \mathrm{a}$, we considered previously manually annotated cell 455 sample, $\beta_{0}$ is the intercept of the model, $X_{z}^{(i)}$ indi- 504 types 28 and included all 14 cell state markers. dif456 cates the $z$-th covariate in the $i$-th sample, $\beta_{z}$ repre- $505 f c y$ t's limma and LMM methods were applied via dif457 sents the coefficient for the $z$-th covariate, and $\epsilon_{c}^{(i)}$ is 506 fcyt's testDS_limma and testDS_LMM functions, re458 the residual for the $c$-th observation in the $i$-th sample. 507 spectively $2 \overline{28}$. We accounted for the paired design by ${ }_{459}$ We infer parameters $\beta_{0}, \ldots, \beta_{Z}$ via least squares regres- 508 modelling the patient id as a covariate.

460 sion, with the estimated values denoted by $\hat{\beta}_{0}, \ldots, \hat{\beta}_{Z}$.

461 We then remove the estimated effect of covariates as $462 y_{c}^{(i)}-\sum_{z=1}^{Z} \hat{\beta}_{z} X_{z}^{(i)}$; differential testing is performed, as 463 described above, on these normalized values. For DS 464 analyses, model (3) is fit, separately, for every gene465 cluster combination, hence accommodating for cell-type 466 specific effects of covariates.

\section{Normalization}

468 In scRNA-seq datasets, CPMs and $\log 2$-CPMs were 517 pression mean greater than 0.2; for DB patterns, we 469 computed via scater Bioconductor $\mathrm{R}$ package [16], 518 increased this threshold to 1 because with low expres470 while vstresiduals were calculated via sctransform $\mathrm{R} 519$ sion values differences are not visible by eye. For every

\section{9 muscat simulation and Kang data}

510 In all muscat simulations, we used the control samples 511 of the Kang dataset as a anchor data; as in the real 512 data analyses, we excluded one sample as it emerged 513 as a potential outlier (Supplementary Figures 5-7), and 514 only considered singlet cells and cells assigned to a cell 515 population. In muscat's simulation studies, we con516 sidered gene-cluster combinations with simulated ex- 
bioRxiv preprint doi: https://doi.org/10.1101/2020.11.24.394213; this version posted May 6, 2021. The copyright holder for this preprint (which was not certified by peer review) is the author/funder, who has granted bioRxiv a license to display the preprint in perpetuity. It is made available under aCC-BY 4.0 International license.

520 simulations, five replicates were simulated, and results 567 521 were averaged across replicates. In the main simulation 568 522 (Figure 2) and the batch effect simulation (Supplemen- 569 523 tary Figure 3), we simulated from a paired design $2^{57}$ 524 groups of 3 samples each, with 4,000 genes, and 3,600 572 525 cells distributed in 3 clusters (corresponding to an av- 574 526 erage of 200 cells per sample in each cluster). For the 575 527 simulation study when varying the number of cells (Fig- 576 528 ure 5 and Supplementary Figure 3), the total numbers 529 of available cells were $900,1,800,3,600$ and 7,200, cor530 responding to an average of 50, 100, 200 and 400 cells 531 per sample in every cluster. For the differential sim- ${ }_{5}^{5}$ 532 ulations, we used $\log 2-\mathrm{FC}$ values of 1 for DE, 1.5 for $533 \mathrm{DP}$ and DM, and 3 for DB and DV. For the batch ${ }_{585}^{58}$ 534 effect simulation study we used a modified version of 535 muscat, developed by Almut Luetge at the Robinson 536 lab (available at: https://github.com/SimoneTiberi/ 537 distinct_manuscript), which allows simulating cluster${ }_{538}$ specific batch effects [5, 14]. All muscat simulation stud- ${ }_{559}^{59}$ 539 ies, as well as the Kang non-null data analysis, were ${ }_{595}^{594}$ 540 performed by editing the original snakemake workflow 541 from Crowell et al. [5]. PB methods were applied on 542 aggregated data by summing cell-level measurements; 543 for differential testing, we used muscat's $p b D S$ function 544 [5]. Mixed model methods were implemented, via mus545 cat's $m m D S$ function, using the same approaches as in ${ }_{546}$ Crowell et al. [5]: in MM-dream2 and MM-vstresiduals 547 linear mixed models were applied to log-normalized 606 548 data with observational weights and variance-stabilized ${ }_{600}^{60}$ 549 data, respectively, while in MM-nbinom generalized lin550 ear mixed models were fitted directly to raw counts. In ${ }_{611}^{610}$ 551 the muscat simulations and in the Kang non-null data 552 analysis, we accounted for the paired design by mod553 elling the patient id as a covariate in all methods that 554 allow for covariates (i.e., distinct, $\mathrm{PB}$ and MM).

\section{${ }_{555} \mathrm{P}$-values adjustment}

556 All p-values were adjusted via Benjamini-Hochberg cor557 rection [2]. In diffcyt simulations we used globally ad558 justed p-values for all methods, i.e., p-values from all 559 clusters are jointly adjusted once. However, since PB 560 methods were found to be over-conservative when glob561 ally adjusting p-values [5], in muscat simulations and 562 Kang discovery analyses, we used locally adjusted p563 values for all methods.

\section{${ }_{564}$ Software versions}

565 All analyses were performed via $\mathrm{R}$ software version 566 4.0.0, with Bioconductor packages from release 3.11.

\section{References}

[1] R. A. Amezquita, A. T. Lun, E. Becht, V. J. Carey, L. N. Carpp L. Geistlinger, F. Marini, K. Rue-Albrecht, D. Risso, C. Soneson, et al. Orchestrating single-cell analysis with bioconductor. Nature methods, $17(2): 137-145,2020$.

[2] Y. Benjamini and Y. Hochberg. Controlling the false discovery rate: a practical and powerful approach to multiple testing. Journal of the Royal statistical society: series B (Methodological), 57(1):289-300, 1995.

[3] B. Bodenmiller, E. R. Zunder, R. Finck, T. J. Chen, E. S. Savig, R. V. Bruggner, E. F. Simonds, S. C. Bendall, K. Sachs, P. O. Krutzik, et al Multiplexed mass cytometry profiling of cellular states perturbed by smallmolecule regulators. Nature biotechnology, 30(9):858-867, 2012.

[4] H. L. Crowell. muscData: Multi-sample multi-group scRNA-seq data 2020. R package version 1.1.2.

[5] H. L. Crowell, C. Soneson, P.-L. Germain, D. Calini, L. Collin, C. Raposo, D. Malhotra, and M. D. Robinson. muscat detects subpopulationspecific state transitions from multi-sample multi-condition single-cell transcriptomics data. Nature Communications, 11(1):1-12, 2020.

[6] G. Csardi and T. Nepusz. The igraph software package for complex network research. InterJournal, Complex Systems:1695, 2006.

[7] M. R. Doherty, H. Cheon, D. J. Junk, S. Vinayak, V. Varadan, M. L. Telli, J. M. Ford, G. R. Stark, and M. W. Jackson. Interferon-beta represses cancer stem cell properties in triple-negative breast cancer. Proceedings of the National Academy of Sciences, 114(52):13792-13797, 2017.

[8] G. Finak, A. McDavid, M. Yajima, J. Deng, V. Gersuk, A. K. Shalek, C. K. Slichter, H. W. Miller, M. J. McElrath, M. Prlic, et al. MAST: a flexible statistical framework for assessing transcriptional changes and characterizing heterogeneity in single-cell RNA sequencing data. Genome biology, 16(1):1$13,2015$.

[9] C. Hafemeister and R. Satija. Normalization and variance stabilization of single-cell RNA-seq data using regularized negative binomial regression. Genome biology, 20(1):1-15, 2019.

[10] H. M. Kang, M. Subramaniam, S. Targ, M. Nguyen, L. Maliskova, E. McCarthy, E. Wan, S. Wong, L. Byrnes, C. M. Lanata, et al. Multiplexed droplet single-cell RNA-sequencing using natural genetic variation. Nature biotechnology, 36(1):89, 2018.

[11] P. V. Kharchenko, L. Silberstein, and D. T. Scadden. Bayesian approach to single-cell differential expression analysis. Nature methods, 11(7):740-742, 2014

[12] K. D. Korthauer, L.-F. Chu, M. A. Newton, Y. Li, J. Thomson, R. Stewart, and C. Kendziorski. A statistical approach for identifying differential distributions in single-cell RNA-seq experiments. Genome biology, 17(1):222 2016

13] M. I. Love, W. Huber, and S. Anders. Moderated estimation of fold change and dispersion for RNA-seq data with DESeq2. Genome biology, 15(12):550, 2014 .

[14] A. Lütge, J. Zyprych-Walczak, U. B. Kunzmann, H. L. Crowell, D. Calini D. Malhotra, C. Soneson, and M. D. Robinson. Cellmixs: quantifying and visualizing batch effects in single-cell rna-seq data. Life science alliance, $4(6), 2021$.

[15] F. J. Massey Jr. The kolmogorov-smirnov test for goodness of fit. Journal of the American statistical Association, 46(253):68-78, 1951.

619 [16] D. J. McCarthy, K. R. Campbell, A. T. Lun, and Q. F. Wills. Scater pre-processing, quality control, normalization and visualization of single-cel 621 RNA-seq data in R. Bioinformatics, 33(8):1179-1186, 2017.

7] M. Nowicka, C. Krieg, L. M. Weber, F. J. Hartmann, S. Guglietta, B. Becher, M. P. Levesque, and M. D. Robinson. CyTOF workflow: differential discovery in high-throughput high-dimensional cytometry datasets. F1000Research, 6, 2017.

8] B. Phipson and G. K. Smyth. Permutation p-values should never be zero: Calculating exact p-values when permutations are randomly drawn. Statistical applications in genetics and molecular biology, 9:Article39, 2010.

29 [19] X.-Q. Qin, N. Tao, A. Dergay, P. Moy, S. Fawell, A. Davis, J. M. Wilson, and $\mathrm{J}$. Barsoum. Interferon- $\beta$ gene therapy inhibits tumor formation and causes regression of established tumors in immune-deficient mice. Proceedings of the National Academy of Sciences, 95(24):14411-14416, 1998.

22] C. Soneson and M. D. Robinson. Bias, robustness and scalability in singlecell differential expression analysis. Nature methods, 15(4):255, 2018.

J. W. Squair, M. Gautier, C. Kathe, M. A. Anderson, N. D. James, T. H Hutson, R. Hudelle, T. Qaiser, K. J. Matson, Q. Barraud, et al. Confronting false discoveries in single-cell differential expression. bioRxiv, 2021. 
bioRxiv preprint doi: https://doi.org/10.1101/2020.11.24.394213; this version posted May 6, 2021. The copyright holder for this preprint (which was not certified by peer review) is the author/funder, who has granted bioRxiv a license to display the preprint in perpetuity. It is made available under aCC-BY 4.0 International license.

[24] P.-Y. Tung, J. D. Blischak, C. J. Hsiao, D. A. Knowles, J. E. Burnett, J. K. Pritchard, and Y. Gilad. Batch effects and the effective design of single-cell gene expression studies. Scientific reports, 7:39921, 2017.

[25] M. Uhlén, L. Fagerberg, B. M. Hallström, C. Lindskog, P. Oksvold, A. Mardinoglu, A. Sivertsson, C. Kampf, E. Sjöstedt, A. Asplund, et al. Tissue-based map of the human proteome. Science, 347(6220), 2015.

[26] C. A. Vallejos, J. C. Marioni, and S. Richardson. BASiCS: Bayesian analysis of single-cell sequencing data. PLoS Comput Biol, 11(6):e1004333, 2015.

[27] T. Wang, B. Li, C. E. Nelson, and S. Nabavi. Comparative analysis of dif653 ferential gene expression analysis tools for single-cell RNA sequencing data. $654 \quad B M C$ bioinformatics, 20(1):40, 2019

55 [28] L. M. Weber, M. Nowicka, C. Soneson, and M. D. Robinson. diffcyt: Differential discovery in high-dimensional cytometry via high-resolution clustering. Communications biology, 2(1):1-11, 2019.

[29] L. M. Weber and C. Soneson. Hdcytodata: Collection of highdimensional cytometry benchmark datasets in bioconductor object formats. F1000Research, 8, 2019 .

[30] Y. Zhang, L. Zheng, L. Zhang, X. Hu, X. Ren, and Z. Zhang. Deep single-cell RNA sequencing data of individual $\mathrm{T}$ cells from treatment-naive colorectal cancer patients. Scientific data, 6(1):1-15, 2019 